\title{
COMPARISON OF AUTO-ASSOCIATIVE MODELS BASED SENSOR COMPENSATION METHODS APPLIED FOR FAULT TOLERANT OPERATION IN MOTOR DRIVES
}

\author{
Luigi Galotto $^{1}$, João O. P. Pinto ${ }^{1}$, Luciana C. Leite ${ }^{1}$, Luiz E. B. Silva ${ }^{2}$, Burak Ozpineci ${ }^{3}$, Bimal K. Bose $^{4}$ \\ ${ }^{1}$ Universidade Federal de Mato Grosso do Sul, Campo Grande, MS, Brazil \\ ${ }^{2}$ Federal University of Itajubá, Itajubá, MG, Brazil \\ ${ }^{3}$ Oak Ridge National Lab, Oak Ridge, TN, USA \\ ${ }^{4}$ The University of Tennessee, Knoxville - TN - USA \\ luigi@batlab.ufms.br, jpinto@nin.ufms.br, lcleite@del.ufms.br
}

\begin{abstract}
Several approaches related to fault tolerant motor control have already been proposed. However, most of them consider the sensors fault-free and work about faults in motors and actuators. Sensors are the fundamentals in any feedback control system. The bad calibration of sensors in motor drives may lead to degradation of performance and even to instability. The purpose of this work is to evaluate some models presented in recent publications to perform on-line sensor fault compensation. In a standard fault tolerant approach, the fault would be detected and the sensor would be isolated. The faulted sensor may have an off-set or scaling error and could still be used if its error is compensated. In this paper, different mathematical solution based on auto-associative models will be evaluated and compared. This technique is described and applied in indirect vector control of an induction motor. Simulated and experimental results are discussed.
\end{abstract}

Keywords - Drive Systems, Auto-associative Models, Sensor drift compensation, Induction Motor.

\section{INTRODUCTION}

The importance of motor drives with vector control is already highlighted in the word. Vector controlled induction motor drives have replaced other expensive machines with comparable performance requirements. However, it is well known that the performance is completely dependent on the measurements and fault in sensors may lead to instability. Therefore, it is mandatory to have a solution for sensor fault compensation in motor drives applied to critical processes.

Despite the importance of sensors in motor drives, this subject is relatively recent and there are only a few published papers about faults in sensors and its compensation in motor drives [1-6]. There are several approaches to fault tolerance in motor control [7-10], but most of them are aimed at faults on power semiconductors and on motors.

The scaling error (multiplicative) in current measurement causes oscillations in the torque at the same electrical frequency and the off-set error (additive) in current causes oscillations in the torque with the double electrical frequency [4]. Faults in a position sensor in rotor may result in overcurrents with heavy load or high speed of operation due to

Manuscript received 31/10/10; first review 19/07/11; second review 27/08/11. Accepted for publication 27/08/2011 recommended for the special section by the editor in charge Antonio J. Marques Cardoso. the decoupling failure between the $\mathrm{d}$ and $\mathrm{q}$ axes. Under light load and low speed, the torque linearity would not be kept [5]. The error in voltage sensors results in problems with PI controllers tuning and in the decoupling term of the counterelectromotive force in the current controller [5]. Only the fault in current sensors is evaluated here in order to introduce the methodology, but the same principle can also be applied to the voltage sensors. Also, any other sensors such as temperature, vibration and flux can be used with this technique.

In [4], the current sensors are compensated using the estimation of the off-set or scaling error based on the speed measurements. The current errors are well compensated, but the speed sensor is also susceptible to faults.

In [5], the current, voltage, and speed sensors are considered. Every sensor is estimated as a function of the others and if the encoder is faulted, the controller is switched to the sensorless mode.

In [6], the fault detection is done using the sum of the three currents. If it is not zero, the sensor with the highest peak is disconnected. The same procedure is applied to the voltage sensors.

It is important to note that the compensation is more desirable than the detection and isolation of the sensors. Isolate will always imply in the open loop control unless there are redundant sensors. The sensor compensation found in literature is always based on the other sensor measurements, which may also be faulty. This is the challenge of the sensors compensation.

Strategies based on auto-associative model (AAM) techniques have been successfully applied for sensor validation and fault detection in huge processes such as nuclear power plants and chemical plants. With this approach a sensor may be sent to maintenance only when a fault is detected. The estimation of the auto-associative models has important features, such as noise reduction, improvement of the accuracy and fault rejection. In other words, the estimates are more reliable than the measurements and can be used to compensate the sensor errors in the feedback control. This approach, have recently be showed in previous works [1-3] and will be deeply explored in this paper.

The aim of this paper is make a comparison of the previously reported methods for this specific application. Also, it will be shown how to use the performance metrics to evaluate the methods for other applications. Moreover, three other AAM sensors compensation in motor drives will be evaluated: (Linear, MSET and Partial Linear). 


\section{MOTOR DRIVER OVERVIEW}

Figure 1 shows the block diagram of induction motor drive system using stator flux-oriented indirect vector control. The stator flux orientation has the advantage that its parameter variation problem is less compared to that of rotor flux orientation. The power circuit in the figure consists of a DC source (battery or rectifier DC), PWM IGBT inverter and cage type induction motor. The Figure 2 shows how the AAM is included in the conventional control scheme, comparing with Figure 1. The signal processing blocks include machine phase current sensors, auto-associative model based drift compensator, signals computation and controller, and the PWM algorithm as shown. The command torque $\left(\mathrm{T}_{\mathrm{e}}{ }^{*}\right)$ and stator flux $\left(\psi_{\mathrm{s}}{ }^{*}\right)$ generate the active $\left(\mathrm{i}_{\mathrm{qs}}{ }^{*}\right)$ and reactive $\left(\mathrm{i}_{\mathrm{ds}}{ }^{*}\right)$ current commands within the block which are then translated into command voltage $\left(\mathrm{V}^{*}\right)$ and its angular position $\left(\theta^{*}\right)$ through the unit vector generated by the speed encoder output $\left(\omega_{\mathrm{r}}\right)$ and feedforward slip frequency. The feedback stator flux is calculated with the current model using speed and feedback currents. One reason for selecting this drive topology was that the setup already existed in the laboratory for the project. However, the approach proposed here can also be applied to other drive control schemes.

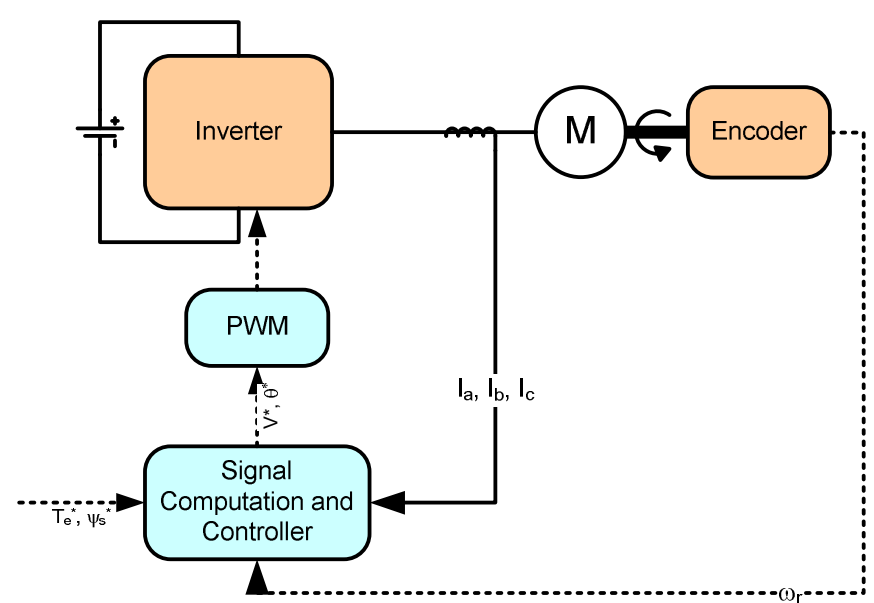

Fig. 1. Block diagram of stator flux oriented vector-controlled drive.

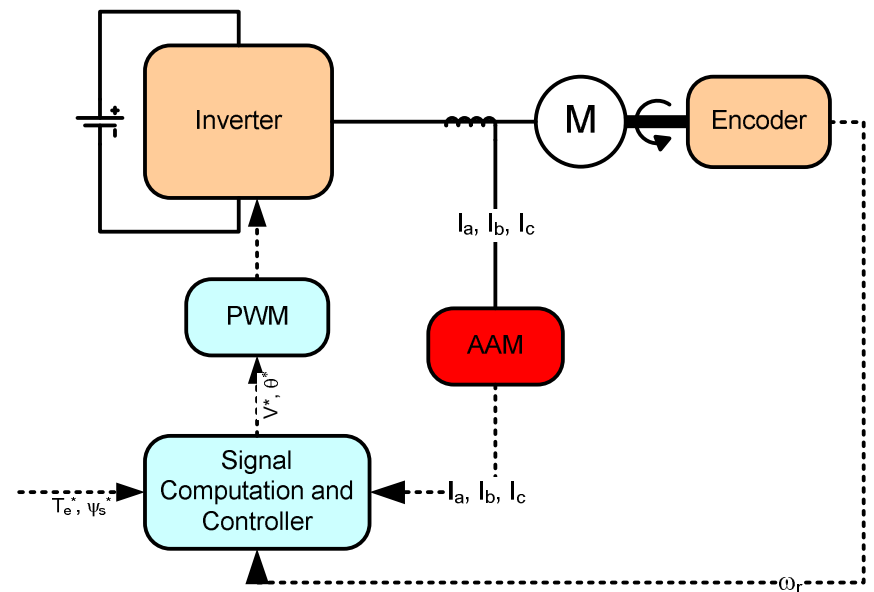

Fig. 2. Block diagram of a stator flux oriented vector-controlled drive using AAM Drift Compensation of current sensors.

\section{EFFECTS OF SENSOR FAULT IN FEEDBACK CONTROL}

Consider the dynamic system represented in the statespace equations (1). In (1), $\boldsymbol{x}$ is the vector of states, $\boldsymbol{u}$ is the vector of inputs and $\boldsymbol{y}$ is the vector of outputs. $\boldsymbol{A}, \boldsymbol{B}$ and $\boldsymbol{C}$ are matrices that define the dynamic behavior of the process. The first equation is the state-space equation and the second is known as state observer neglecting the influence of $\boldsymbol{u}$ in $\boldsymbol{y}$.

$$
\left\{\begin{array}{c}
\dot{x}=A \cdot x+B \cdot u \\
y=C \cdot x
\end{array}\right.
$$

Equation (2) shows the equivalent state-space equations of the system of (1) in closed loop considering $\boldsymbol{u}=\boldsymbol{r} \boldsymbol{- y}$, where $\mathrm{r}$ is the reference and input in closed loop.

$$
\dot{\boldsymbol{x}}=(\boldsymbol{A}-\boldsymbol{B} \cdot \boldsymbol{C}) \cdot \boldsymbol{x}-\boldsymbol{B} \cdot \boldsymbol{r}
$$

The matrix that multiplies $\mathrm{x}$ determines the dynamic features of the system, including controllability and stability. The equations with additive error, such as an offset in the sensor measurement, in (3) are calculated using $\boldsymbol{u}=\boldsymbol{r} \boldsymbol{-}(\boldsymbol{y}+$ $\boldsymbol{e})$, where $\mathrm{e}$ is an error.

$$
\dot{\boldsymbol{x}}=(\boldsymbol{A}-\boldsymbol{B} \cdot \boldsymbol{C}) \cdot \boldsymbol{x}+\boldsymbol{B} \cdot(\boldsymbol{r}-\boldsymbol{e})
$$

Equation (3) shows that there is no change in the dynamics of the system. However, the consequence of the additive error will be the change of the point of operation of the system. In (4), the state-space equations are obtained with a multiplicative error, such as attenuation of excessive gain in the sensors signal conditioning, considering $\boldsymbol{u}=\boldsymbol{r} \boldsymbol{- y} \boldsymbol{e}$.

$$
\dot{\boldsymbol{x}}=(\boldsymbol{A}-\boldsymbol{B} \cdot \boldsymbol{C} \cdot \boldsymbol{e}) \cdot \boldsymbol{x}-\boldsymbol{B} \cdot \boldsymbol{r}
$$

This error is equivalent to a feedback gain and it changes the dynamics and the stability of the system. In this point of view, multiplicative errors are more critical than additive errors, but both of them can lead to undesirable effects accordingly their intensity.

Multiplicative or additive errors may be produced by faults in sensors due to aging, interference, physical damage, and others. The AAM is successful, because it reduces the magnitude of the error in the presence of redundancy as explained in the next sections.

\section{A. Stability}

Evaluating the stability for linear state space models, it is well known that it is dependent only on the eigenvalues of the state matrix (matrix that multiply the state vector $\boldsymbol{x}$ ). In equation (3), the additive error does not change the state matrix in closed loop and it has an effect of change the referent $\boldsymbol{r}$. On the other hand, multiplicative error calibration changes the state matrix, as shown in equation (4), and it may turn the system more susceptible to instability.

In fact, the real system is not ideally linear and even additive fault may cause stability problems. But this notion of stability, observing the state matrices in (3) and (4), shows that the fault effects of multiplicative errors may be usually severer than the effects additive errors. 


\section{STUDY OF THE FAULT EFFECTS}

The sensor drift compensation AAM was studied initially on MATLAB/Simulink simulation with a $20 \mathrm{hp}$ drive system. In the simulated drive system, the current measurement in one phase was injected with additive (1.5 A or $15 \%$ of the nominal current) and multiplicative (with a factor of 0.05) errors, whereas the other two phase currents remain normal. Then the drive system was tested without compensation. Figure 3 shows the resulting speed response, where the set speed, actual response without fault and that with fault condition are indicated. Obviously, speed oscillation is induced by severe unbalance in the phase currents. It is important to note that three current sensors are used in this case and the fault is applied only in one sensor. In normal application with two sensors, the fault in one sensor is extended for two phases and the resulting fault effect is worse than that shown in Figure 3.

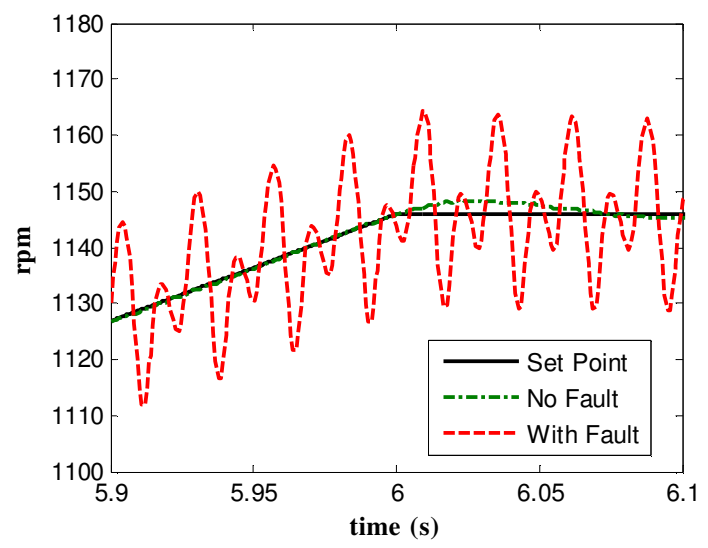

Fig. 3. Simulated current sensor fault effect on speed oscillation.

Therefore, even without compensation, the simple procedure of using three current sensors instead of two helps to improve the performance. Figure 4 shows the frequency components of the speed oscillation. The multiplicative error generates oscillation at double the electrical frequency, whereas the additive error generates oscillation at the system electrical frequency.

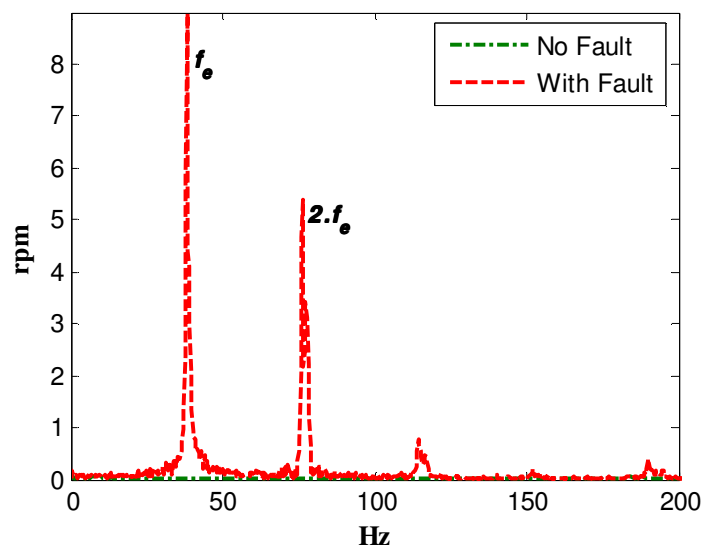

Fig. 4. Simulated fault effect on speed oscillation in frequency domain.
Figure 5 shows the real stator currents in the motor in normal operation (a) and under fault conditions (b). Although there is no fault in the motor or actuator, there are high currents due to the fault in sensor, which may be dangerous to other components.

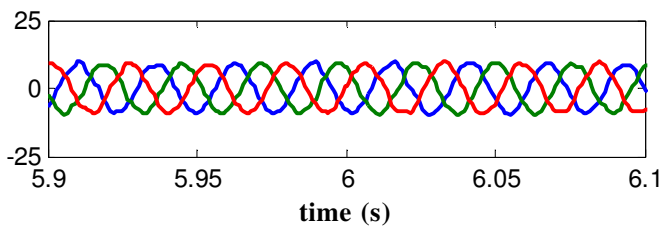

(a)

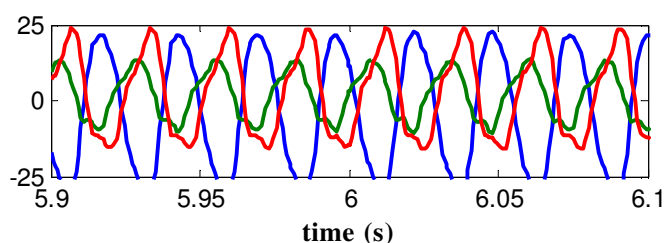

(b)

Fig. 5. Stator Currents for Normal (a) and Fault (b) Conditions.

More than just oscillations have been observed in vector controlled induction motor. Depending on the type and intensity of the error the system may goes unstable, as shown in the simulation of Figure 6 . In this simulation, a multiplicative error was exponentially applied to the system in such way as the initial gain was 1 (no error) and the gain in the imminent instability was about 0,2 .

The Figure 7 shows the fault effect in the control using an AAM. The measured current of phase $A$ suffers deep distortion compared with the real current. On the other hand, the estimated currents are closer to the real currents than the measurements. Once these estimated currents are used in the control, the effects of overcurrents presented in Figure 5 are reduced. Increasing the number of redundant sensors and using different methods this improvement can be even better of this example of compensation.

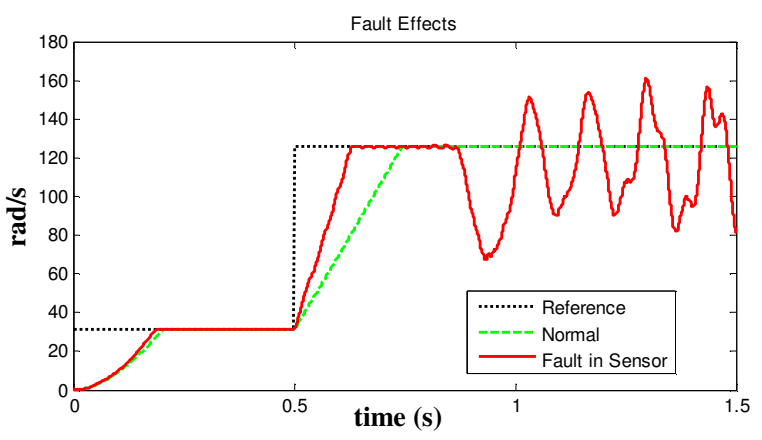

Fig. 6. Instability caused by multiplicative error. 

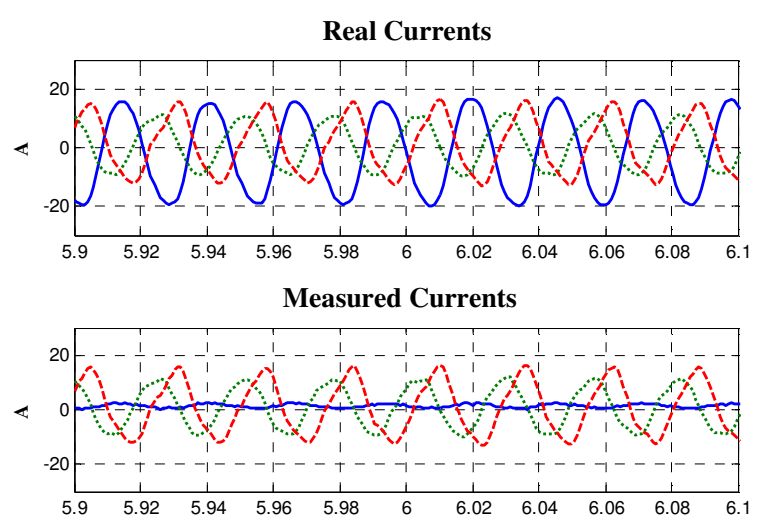

Estimated Currents

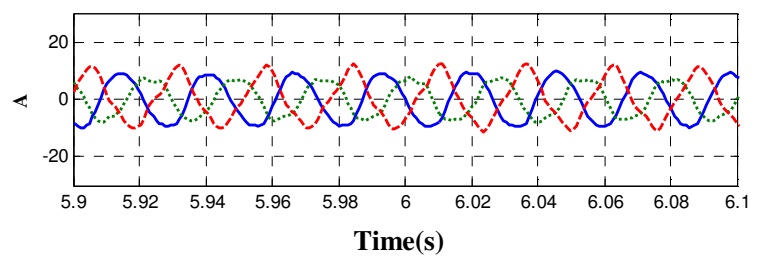

Fig. 7. Currents operating with AANN compensation.

\section{AUTO-ASSOCIATIVE MODELS OVERVIEW}

The auto-associative models can be built using several different techniques, where AANN and AAKR is just one of them. These models have been used in monitoring and diagnosis very often, but just recently they are being used in control loops to compensate the sensor fault effect directly. Therefore, it is not well-known exactly the advantages and drawbacks of these models. The comparisons described in this work aims to overcome this gap and methodology presented may help future evaluation of each technique for each application.

The conventions and performance metrics used to compare the methods will be first described, and then, the comparison will be presented.

\section{A. Conventions}

Consider the generic auto-associative model in Figure 8. The models trained with data (data based or empirical models) usually have a big number of inputs and outputs, because they are used to model complex systems. In the sensors current application, the models have three outputs (because three current values are needed) and the number of inputs is equal to the number of sensors presented in the training data.

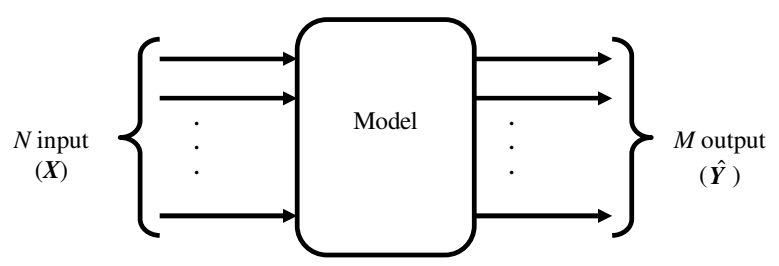

Fig. 8. Generic Auto-Associative Model.
The training is performed off-line so the training data set must be well chosen to make sure that the model will provide good estimates in any operating point.

It is useful a clear definition of the notation used to better understand these models and the performance metrics. Suppose that $\boldsymbol{x}_{\boldsymbol{i}}$ is the $i^{\text {th }}$ of the $N$ inputs and $\boldsymbol{y}_{j}$ is the $j^{\text {th }}$ of the $M$ outputs, accordingly the Figure 8 . And $\hat{\boldsymbol{y}}_{\boldsymbol{j}}$ is the $j^{\text {th }}$ estimation correspondent to the output $\boldsymbol{y}_{\boldsymbol{j}}$. Note that $\boldsymbol{x}_{\boldsymbol{i}}, \boldsymbol{y}_{j}$ and $\hat{\boldsymbol{y}}_{\boldsymbol{j}}$ are bold because they are vectors with $n$ observations each. Also, let is consider $\boldsymbol{X}, \boldsymbol{Y}$ and $\hat{\boldsymbol{Y}}$ as matrices that contain the vectors $\boldsymbol{x}_{\boldsymbol{i}}, \boldsymbol{y}_{\boldsymbol{j}}$ and $\hat{\boldsymbol{y}}_{\boldsymbol{j}}$ respectively. The Figure 9 represents the described notation.
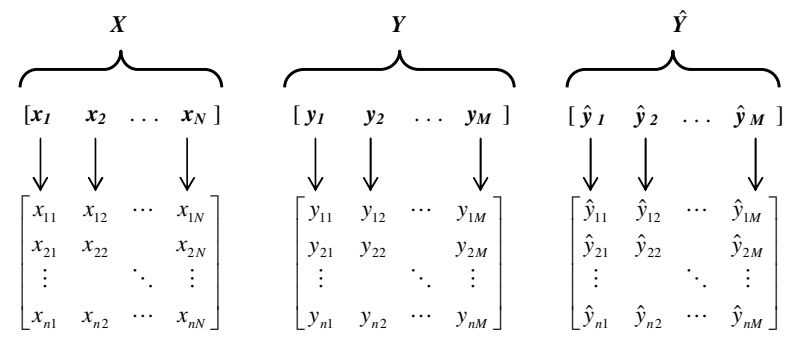

Fig. 9. Notation.

In practice, each column of matrix $\boldsymbol{X}$ represents a sensor used in the input of the model and the columns of matrix $\boldsymbol{Y}$ are signals whose measurements have to be estimated. Both matrix are the same when an estimation of each sensor is needed. The matrices $\boldsymbol{X}$ and $\boldsymbol{Y}$ are the data used to build the model. Matrix $\hat{\boldsymbol{Y}}$ are estimations of $\boldsymbol{Y}$ obtained with the trained model for each input $\boldsymbol{X}$.

\section{B. Performance Metrics}

The quality of each model may be defined using some important performance metrics. Some of them are: mean square error and sensibility. They can show if the model may improve the system response in normal conditions of operation and if the model can improve the response in fault conditions.

The mean square error (MSE) in equation (5), compare the estimated output with the actual output without noise. In (5), $n$ is the number of observations, $\hat{y}_{k j}$ is the $k^{\text {th }}$ estimation of the signal $j$ and $y_{k j}$ is the $k^{\text {th }}$ observation.

$$
M S E_{j}=\frac{1}{n} \sum_{k=1}^{n}\left(\hat{y}_{k j}-y_{k j}\right)^{2}
$$

This metric is well known and is useful to determine the accuracy of the model. This accuracy is fundamental to assure that the control system will keep the same response using the estimations of the model as a feedback signal in the control loop. On the other hand, the sensibility is a metric to define the improvement of the transient response in the presence of fault.

Consider that $x_{k i}^{\text {drift }}$ is the kth observation of xi with artificially generated drift and $\hat{y}_{k j}^{d r i f t}$ is the kth estimate 
observation of yi to the same inputs with the drift. This drift may be additive or multiplicative changing the off-set or the scale of the original signal. Thus, the denominator in equation (6) represents a simulated drift in the input $i$ and the numerator is the propagation of the fault to the estimation of the signal $\mathrm{j}$.

$$
S_{i j}=\frac{\sum_{k=1}^{n}\left|\hat{y}_{k j}^{d r i f t}-\hat{y}_{k j}\right|}{\sum_{k=1}^{n}\left|x_{k i}^{d r i f t}-x_{k i}\right|}
$$

This effect of propagation can also be observed in the illustration in Figure 10. Once an error is simulated in one input, this error is propagated to the all estimations $y$. It happens because each estimation is a combination of all inputs. In ideal condition, the error in any input should not be presented in the estimations.

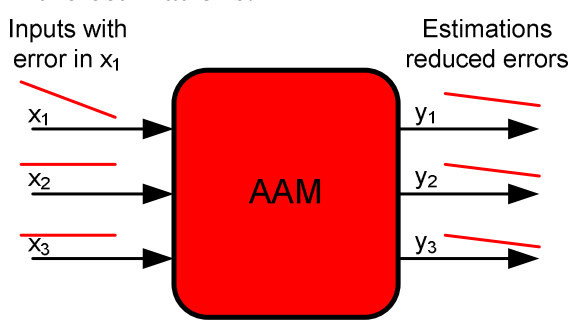

Fig. 10. Illustration of error propagation in AAM.

The result is a matrix which shows the effect in the output $j$ due to a fault in the input $i$. When $i$ is equal to $j$, then it is called robustness. When $i$ is different from $j$, then it is called spillover. The optimum sensibility matrix should be zero so that the fault effects could be completely eliminated.

Equation (7) shows the filtering performance were the noise is rejected from the model. The AAM is no dynamic but it can filter distinct white noise for each sensor since the noises for each sensor are not correlated. That is the reason that allows AAM increases the precision of the measurements.

$$
F_{i j}=\frac{\sum_{k=1}^{n}\left|\hat{y}_{k j}^{n o i s e}-\hat{y}_{k j}\right|}{\sum_{k=1}^{n}\left|x_{k i}^{n o i s e}-x_{k i}\right|}
$$

In practice, this equation is the same of equation (6). The only difference is that a noise is added in each input instead of a constant error.

\section{EXPERIMENTAL RESULTS}

The results have been obtained using and indirect vector controller $1 / 2$ HP induction motor. The motor is controlled to track the speed path indicated by the black line in Figure 11 and Figure 12. The multiplicative and additive faults are applied after 5 seconds of running indicated in Figure 11. The motor vibration is immediate and it loses the stability after 8 seconds. The response in Figure 12 was obtained using the same conditions applied to get the response in
Figure 11, including the same number of sensors and the same fault applied after 5 seconds. The only difference is the use of an AAM in the feedback currents, as presented in [1]. The AAM is implemented on-line as presented in Figure 2 with a $1 \mathrm{~ms}$ of sample time. The vibration was not completely mitigated, but it showed a slightly reduction and the motor kept stable up to the end of the experiment. Obviously, attenuation in speed oscillation occurs due to compensation of the current errors.

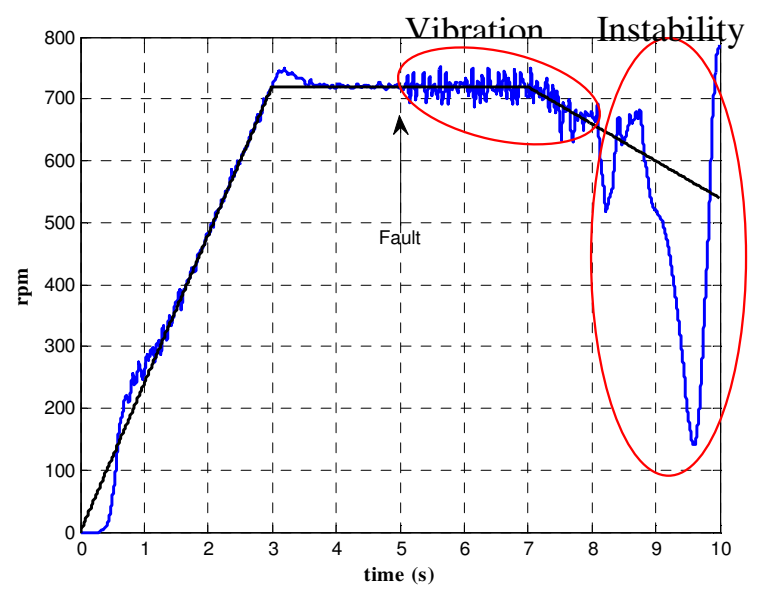

Fig. 11. Experimental current sensor fault effect.

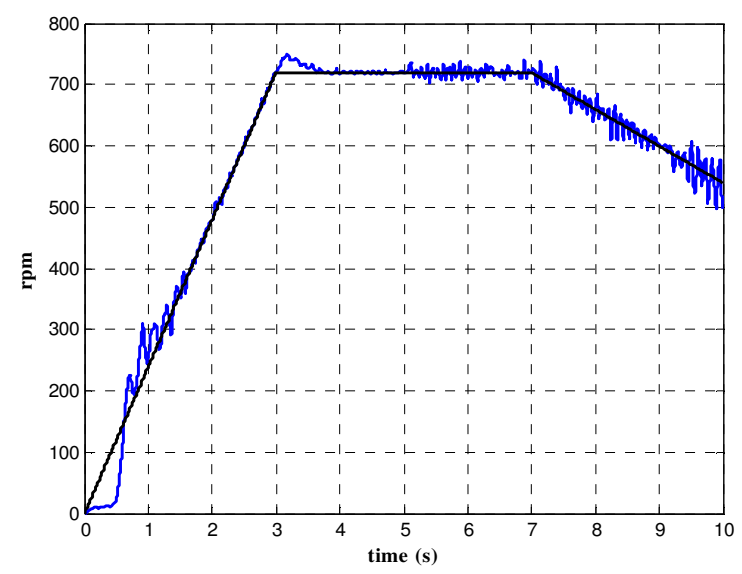

Fig. 12. Experimental current sensor fault effect with AAM compensation.

The fault effect is still observable even with the use of the AAM and it is not possible to assure the system will never go unstable. However, it is possible to confirm that AAM at least delayed the instability and reduced the fault effect. Another important result is that the response was practically not changed in normal operation.

The type of model used to obtain these results was AANN (Auto-Associative Neural Network) which was trained with the features presented in Table I, using simulated data. The trained topology has one more layer than that presented in Figure 2. The bottleneck layer has only two neurons and it is indicated in bold. The performance function considered in the training process is the regularized mean square error. The regularization improves the generalization of the network and its robustness to fault in sensors. 
TABLE I

Main features for the AANN with Three Sensors

\begin{tabular}{ll}
\hline Features & Tested AANN \\
\hline Number of layers & 6 \\
\hline $\begin{array}{l}\text { Number of neurons for each } \\
\text { layer, respectively }\end{array}$ & $3,8,6,2,8$ and 3 \\
\hline Performance Function & Regularized MSE \\
\hline Training Algorithm & Resilient Backpropagation \\
\hline Epochs & 2000 \\
\hline
\end{tabular}

The performance varies with the data set used and for each different AANN trained. Maybe, the high reliability drive systems in the future could have neural networks (specifically trained for each family of drives) used to preprocess all feedback signals from the sensors.

\section{A. Other Results with Kernel Model}

The same experimental setup was used to get results with auto-associative kernel regression (AAKR). This model was made using the same simulated data used for training the AANN. The control is performed to follow the trapezoidal path of speed shown in Figure 13. The motor is accelerated from 0 to 3 seconds up to $900 \mathrm{rpm}$ and decelerated from 7 to 10 seconds. The standard response is compared with the response using the kernel model with three sensors and the kernel model with six sensors.

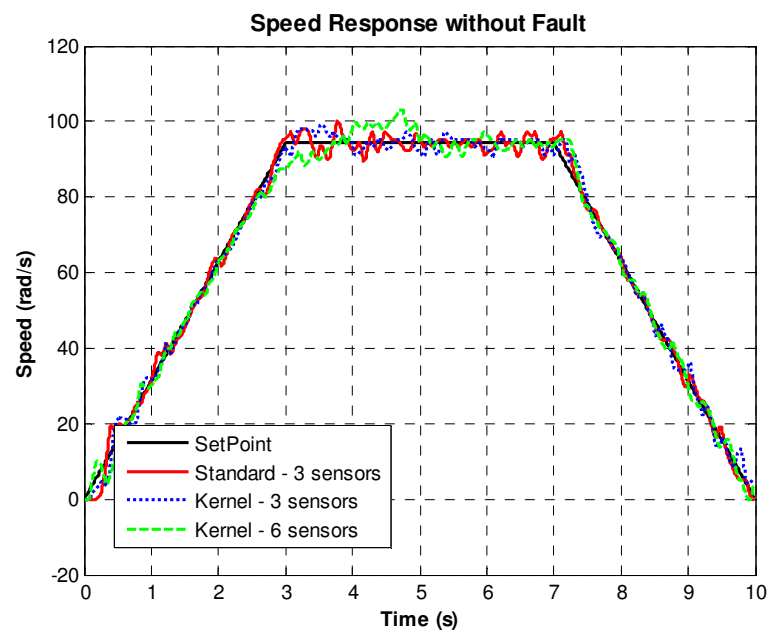

Fig. 13. Experimental Speed Responses without Fault.

The kernel model improves the precision of the measurements and it tends to improve the response also in normal conditions of operation.

A multiplicative fault is added to one current sensor of one phase at 5 seconds after the beginning of the experiment. The results are shown in Figure 14.

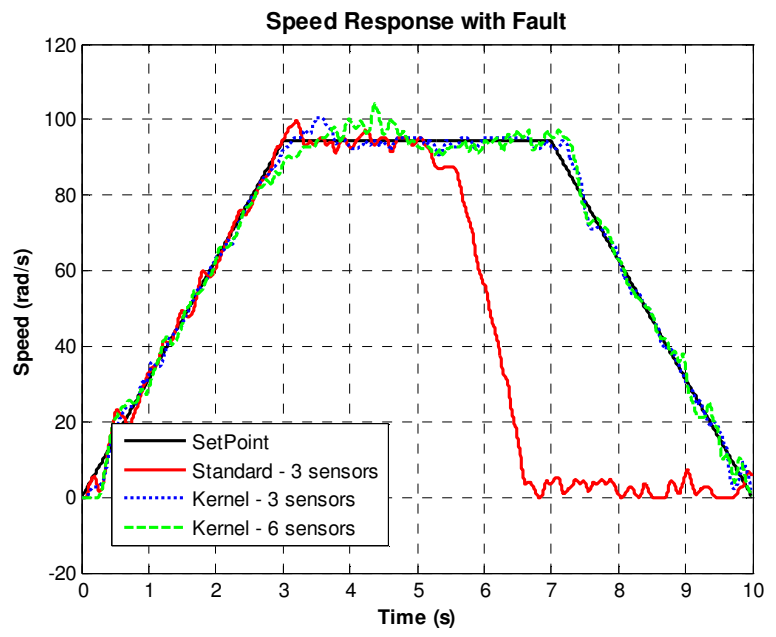

Fig. 14. Experimental Speed Responses with Fault.

In this case, the fault also caused the loss of control. This is due to the gain applied to the current sensor, where the current is amplified. Then, an amplified current is observed by the controller while the actual current is low. As a result, the control action reduces the supposed high current that is actually a low current. It decreases torque and stop the machine.

The kernel models showed to minimize the fault effect and can keep the control even with three sensors [2]. But the use of more sensors could also be useful for multiple faults.

\section{EVALUATION OF THE MODELS}

\section{A. Type of models}

The methods used to create associative models tested in this work were: Linear Regression, Auto-Associative Kernel Regression (AAKR) [2,14], Auto-Associative Neural Network (AANN) [1,11-13], Partial Linear [15] and Multivariate State Estimation Technique (MSET) [16-17]. Also, it has been made the analysis of the performance increasing the number of sensors.

\section{B. Results}

The presented metrics allow the different modeling techniques to be compared.

The tables II, III and IV shows the values of the sensibility matrix found for the linear, kernel and neural network models, respectively, in the application of current compensation. These results were obtained using equation (6). For the linear model, all sensibilities are 0.5 which means that an error in any sensor will affect $50 \%$ all the three estimations (A, B and C).

TABLE II

SENSIBILITY OF A LINEAR MODEL

\begin{tabular}{l|llll}
\hline \multicolumn{1}{c|}{ Propagation } & $A$ & $B$ & $C$ & Sum \\
Drift & & & & \\
\hline Sensor A & 0.5 & 0.5 & 0.5 & 1.5 \\
Sensor B & 0.5 & 0.5 & 0.5 & 1.5 \\
Sensor C & 0.5 & 0.5 & 0.5 & 1.5 \\
\hline
\end{tabular}


The kernel sensibility matrix in Table III is slightly asymmetric and the propagation is not the same for each sensor. This asymmetry is even more intense in the AANN model in Table IV. This difference of these models in relation to the linear model is not necessarily a drawback. It means that the each sensor will be not equally compensated.

The best sensibility matrix should be a zero matrix, which means that no drift in the sensors will be reflected in the estimations.

TABLE III

Sensibility of a Kernel Model

\begin{tabular}{|c|c|c|c|c|}
\hline Propagation & $A$ & $B$ & $C$ & Sum \\
\hline Sensor A & 0.6547 & 0.3240 & 0.3307 & 1.3094 \\
\hline Sensor B & 0.3320 & 0.6592 & 0.3273 & 1.3185 \\
\hline Sensor C & 0.3351 & 0.3230 & 0.6581 & 1.3162 \\
\hline
\end{tabular}

Although some values of propagation are bigger than the other models, the AANN presented some of the smallest values as occurred with the drift in the sensor A. In this case, the model is adequate is this sensor has more probability to be faulted.

TABLE IV

Sensibility of a AANN Model

\begin{tabular}{l|lllll}
\hline Prift & & & & & \\
\hline Sensoragation A & $A$ & $B$ & $C$ & Sum \\
Sensor B & 0.5033 & 0.2732 & 0.2302 & 1.0067 \\
Sensor C & 0.4967 & 0.7268 & 0.2302 & 1.4537 \\
\hline
\end{tabular}

It is important to note that the AANN and the kernel model have similar error compensation. But the AANN is safer to use in normal operation due the zero MSE, shown in Table VI.

These are results of 3 sensors and two models. To show briefly all evaluated results with different models and number of sensors, the Tables V, VI and VII are presented. The propagated error in Table $\mathrm{V}$ is calculated with the average of the sum column presented in Tables II, III and IV. Then, this percentage number reflects the capability of the model to reject error. All models with 2 sensors presented twice the input error. Independent of the model used. That is because there is not redundancy. Increasing the number of sensors de physical and analytical redundancy increases and the propagated error may be under $100 \%$.

TABLE V

Propagated Errors

\begin{tabular}{l|lllll}
\hline $\begin{array}{l}\text { Number of } \\
\text { Current } \\
\text { Sensors }\end{array}$ & 2 & 3 & 4 & 5 & 6 \\
\hline Linear & $200 \%$ & $150 \%$ & $108 \%$ & $86 \%$ & $75 \%$ \\
AAKR & $200 \%$ & $132 \%$ & $99 \%$ & $79 \%$ & $66 \%$ \\
MSET & $200 \%$ & $132 \%$ & $99 \%$ & $79 \%$ & $65 \%$ \\
AANN & $200 \%$ & $133 \%$ & $100 \%$ & $89 \%$ & $82 \%$ \\
Partial & $200 \%$ & $150 \%$ & $100 \%$ & $23 \%$ & $18 \%$ \\
Linear & & & & & \\
\hline
\end{tabular}

The Figure 15 shows graphically the results of Table V.

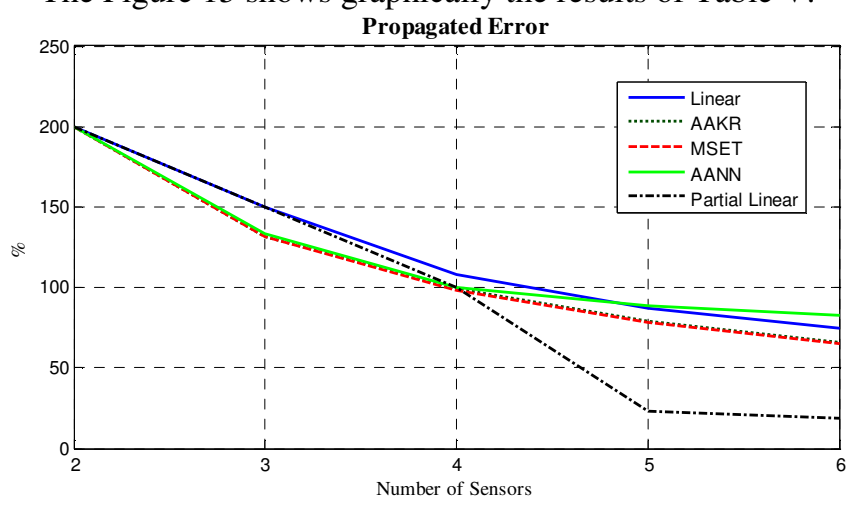

Fig. 15. Propagated Error.

The results of MSE in Table VI are related with the interference of the model in normal operation. Despite the best results of error propagation with 3 or 4 sensors, the AAKR changes the true current measurement even without fault. The impact on the performance still needs to be analyzed to verify if they are acceptable.

TABLE VI

MSE

\begin{tabular}{l|lllll}
\hline $\begin{array}{l}\text { Number of } \\
\begin{array}{l}\text { Current } \\
\text { Sensors }\end{array}\end{array}$ & 2 & 3 & 4 & 5 & 6 \\
\hline Linear & 0,0000 & 0,0000 & 0,0000 & 0,0000 & 0,0000 \\
AAKR & 0,0263 & 0,4846 & 0,0839 & 0,0395 & 0,0003 \\
MSET & 0,0000 & 0,0000 & 0,0000 & 0,0000 & 0,0000 \\
AANN & 0,0000 & 0,0000 & 0,0000 & 0,0000 & 0,0000 \\
Partial & 0,0000 & 0,0000 & 0,0000 & 0,0000 & 0,0000
\end{tabular}

Linear

For mode than five sensors, the partial linear models are far better with good Sensibility and MSE. But Kernel and MSET are the best for filtering, as shown in Table VII. This table has been obtained in similar way of Table V.

TABLE VII Average Filtering

\begin{tabular}{l|lllll}
\hline $\begin{array}{l}\text { Number of } \\
\begin{array}{l}\text { Current } \\
\text { Sensors }\end{array}\end{array}$ & 2 & 3 & 4 & 5 & 6 \\
\hline Linear & 1,1343 & 0,8638 & 0,7416 & 0,6530 & 0,6107 \\
AAKR & 1,1133 & 0,7487 & 0,7062 & 0,6153 & 0,5764 \\
MSET & 1,1344 & 0,8106 & 0,7250 & 0,6425 & 0,5750 \\
AANN & 1,1343 & 0,8392 & 0,8256 & 0,9468 & 0,9372 \\
Partial & 1,1343 & 0,8638 & 0,8088 & 0,7481 & 0,6445 \\
Linear & & & & & \\
\hline
\end{tabular}

Table VII and Figure 16 show the numerical and graphical results for propagated noise.

The use of two sensors is typical in motor drives in order to reduce costs. However, it amplifies the noise in the measurements and the error propagation, which are normally not observed by the driver designer. 


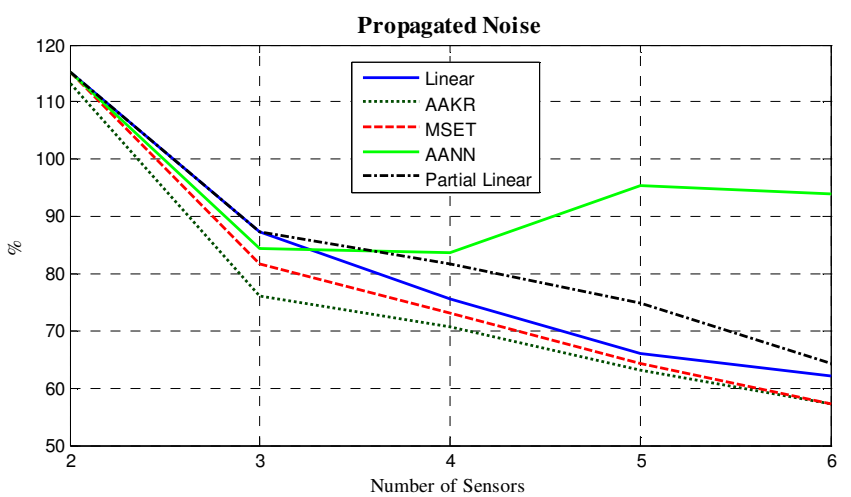

Fig. 16. Propagated Noise.

\section{CONCLUSION}

The current sensor fault compensation has been demonstrated in the paper with different auto-associative models in order to make a comparison of different models in this specific application. An indirect vector-controlled induction motor drive system has been experimentally evaluated to validate the performance improvement in the previous works. However, this is the first work comparing different techniques and their performance. The AAKR and MSET are the best for filtering or noise rejection. The models are not dynamic and the filter effect is due to the noncorrelation of the noise. The filtering metric is also a contribution of this work, which represents the improvement in sensors accuracy. Above five sensors, the partial linear models showed a far best performance in error propagation compared with other techniques. As this is the main metric for fault tolerant operation, it may be preferential to choose the best technique.

The main importance of the presented metrics is that the models can be evaluated before the use in real applications. The principle of operation of the sensors compensation using auto-associative models in feedback control loop showed to reduce the fault effects and let open possible future works with other types of sensors. Multiple fault effects also need to be studied.

\section{REFERENCES}

[1] L. Galotto, B.K. Bose, L.C. Leite, J.O.P. Pinto, L.E.B. da Silva, G.L. Torres, "Auto-Associative Neural Network Based Sensor Drift Compensation in Indirect Vector Controlled Drive System", Proceedings on the Industrial Electronics Society, 2007. IECON 2007. 33rd Annual Conference of the IEEE, pp. 1009-1014, nov, 2007.

[2] L. Galotto, J.O.P. Pinto, B. Ozpineci, L.C. Leite, L.E.B. da Silva, "Sensor Compensation in Motor Drives using Kernel Regression", Proceedings on the Electric Machines \& Drives Conference, 2007. IEMDC '07. IEEE International, vol. 1, pp. 229-234, may, 2007.

[3] L. Galotto, Análise de Compensação De Falta Em Sensores Aplicada Em Controle De Motores, UFMS thesis, Brazil, 2006.

[4] A. El-Antably, L. Xiaogang, R. Martin, "System simulation of fault conditions in the components of the electric drive system of an electric vehicle of an industrial drive", Industrial Electronics, Control, and Instrumentation, 1993. Proceedings of the IECON '93., International Conference on, vol. 2, pp. 1146-1150, 1993.

[5] B.A. Welchko, T.M. Jahns, S. Hiti, "IPM synchronous machine drive response to a single-phase open circuit fault", Power Electronics, IEEE Transactions on, vol. 17, pp. 764-771, 2002.

[6] N. Retiere, D. Roye, and P. Mannevy, "Vector-based investigation of induction motor drive under inverter fault operations", Power Electronics Specialists Conference, 1997. PESC '97 Record., 28th Annual IEEE, vol. 2, pp. 1288-1294, 1997.

[7] R.B. Sepe Jr., B. Fahimi, C. Morrison, J.M. Miller, "Fault-tolerant operation of induction motor drives with automatic controller reconfiguration", Electric Machines and Drives Conference, 2001. IEMDC 2001. IEEE International, pp. 156-162, 2001.

[8] D.W. Chung, S.K. Sul, "Analysis and Compensation of Current Measurement Error in Vector-Controlled AC Motor Drives", Industry Applications, IEEE Transactions on, vol. 34, pp. 340-345, 1998.

[9] Y.S. Jeong, S.K. Sul, S.E. Schultz, N.R. Patel, "Fault Detection and Fault-Tolerant Control of Interior Permanent-Magnet Motor Drive System for Electric Vehicle", Industry Applications, IEEE Transactions on, vol. 41, pp. 46-51, 2005.

[10] S.M. Bennett, R.J. Patton, S. Daley and D.A. Newton, "Torque and Flux Estimation for a Rail Traction System in the Presence of Intermittent Sensor Faults", Control '96, UKACC International Conference on, vol. 1, 72-77, 1996.

[11]M.A. Kramer, "Auto-associative Neural Networks", Computers in Chemical Engineering, vol. 16, No. 4, pp. 313-328, 1992.

[12]M. A. Kramer, "Nonlinear principles component analysis using auto-associative neural network", AIChE Journal, Vol. 37, No. 2, pp. 233-243, 1991.

[13] M.S. Ikbal, H. Misra, B. Yegnanarayana, "Analysis of autoassociative mapping neural networks", in International Joint Conference on Neural Network, 1999.

[14]C.G. Atkeson, A.W. Moorey, S. Schaalz. "Locally Weighted Learning”, Artificial Intelligence Review, vol. 11, pp. 11-73, 1997.

[15]L. Galotto, J.O.P. Pinto, J.W. Hines, R.O. Sanches, B.N. Carrasco, G.S. Tatibana, "Improvement of Fault Detection with Partial Auto-Associative Models", COMADEM, pp. 357-366, 2006.

[16] K.C. Gross, V. Bhardwaj, R. Bickford, "Proactive Detection of Software Aging Mechanisms in Performance Critical Computers", Software Engineering Workshop, 2002. Proceedings. 27th Annual NASA Goddard/IEEE, pp. 17 - 23, 2003.

[17] A.V. Gribok, A.M. Urmanov, J.W. Hines, "Uncertainty Analysis of Memory Based Sensor Validation Techniques", Kluwer Academic Publishers, Real-Time Systems, vol. 27 Issue 1, may 2004. 


\section{BIOGRAPHIES}

Luigi Galotto Jr was born in São Paulo, Brazil in 1981. Bachelors degree in 2003 of electrical engineering at the Federal University of Mato Grosso do Sul (UFMS), in Campo Grande, Brazil. The masters degree on Artificial Intelligent Applications was got in 2006 with the study of sensor fault tolerant operation in drive systems. Currently is finishing doctors at Paulista State University (UNESP) in power electronics.

$\mathrm{He}$ has also been working as a researcher at an acknowledged laboratory at UFMS and developing projects in sprectrum analyzers, power converters, condition monitoring software and control systems.

João Onofre Pereira Pinto was Born in june 22, 1966 in Valparaíso, S.P., Brazil. Electrical Engineer by Paulista State University (UNESP), Ilha Solteira, Brazil, in 1990. Masters in electrical engineering by Federal University of Uberlândia, Uberlândia, Brazil, in 1993, and PhD. by The University of Tennessee, Knoxville, TN, EUA in 2001.

Currently, he is professor of Federal University of Mato Grosso do Sul, Campo Grande, Brazil. Researcher of CNPq, your interest areas includes data mining, decision support systems, signal processing, artificial neural network application, fuzzy logic, genetic algorithms and wavelets in power electronics, PWM techniques, control and electrical machine drives.

Luciana Cambraia Leite has bachelors degree in Electrical Engineering by the Júlio de Mesquita Filho Paulista State University (1993), masters in Electrical Engineering by Federal University of Uberlândia (1997) e Doctors in Electrical Engineering by State University of Campinas (2003). Currently, she is adjunct professor of the Department of Electrical Engeneering and Coordinator of the graduation program in Electrical Engeneering (PPGEE) of Federal University of Mato Grosso do Sul. Your acting areas are related with the following themes: electrical machine drives, application of artificial intelligence techniques in engineering and energetic efficiency.

Luiz Eduardo Borges da Silva graduate at Electrical Engineering from Federal University of Itajubá (1977), master's at Electric Engineering from Federal University of Itajubá (1982) and PhD. in Electrical Engineering from Ecole Polytechnique of Montreal (1988). Has experience in Electric Engineering, focusing on Industrial Electronics, Electronic Systems and Controls, acting on the following subjects: intelligent control, power electronics, system operation, adaptive control and predictive maintenance.

Burak Ozpineci received the B.S. degree in electrical engineering from the Middle East Technical University, Ankara, Turkey, in 1994, and the M.S. and Ph.D. degrees in electrical engineering from the University of Tennessee, Knoxville, in 1998 and 2002, respectively. He joined the Post-Masters Program with the Power Electronics and Electric Machinery Research Center, Oak Ridge National Laboratory (ORNL), Knoxville, TN, in 2001 and became a Full-Time Research and Development Staff Member in 2002 and the Group Leader of the Power and Energy Systems Group in 2008. Presently, he also has an Adjunct Faculty appointment with the University of Arkansas, Fayetteville. $\mathrm{He}$ is doing research on the system-level impact of $\mathrm{SiC}$ power devices, multilevel inverters, power converters for distributed energy resources and hybrid electric vehicles, and intelligent control applications for power converters.

Bimal K. Bose (Life Fellow, IEEE) held the Condra Chair of Excellence (Endowed Chair Professor) in Power Electronics at the University of Tennessee, Knoxville, since 1987, where he was responsible for teaching and the research program in power electronics and motor drives. Concurrently, he was the Distinguished Scientist (19892000) and the Chief Scientist (1987-1989) of EPRI-Power Electronics Applications Center, Knoxville, TN. Prior to this, he was a Research Engineer in the General Electric Corporate Research and Development Center (now GE Global Research Center), Schenectady, NY, for 11 years (1976-1987), an Associate Professor of Electrical Engineering, Rensselaer Polytechnic Institute, Troy, NY for five years (1971-1976), and a faculty member at IIEST (formerly Bengal Engineering and Science University (BESU)) for 11 years (1960-1971). 